7 - ORIGINAL ARTICLE

ISCHEMIA-REPERFUSION

\title{
Expression of FAS ligand in the ipsilateral and contralateral testicles of rats subjected to the torsion of the unilateral testicular cord ${ }^{1}$
}

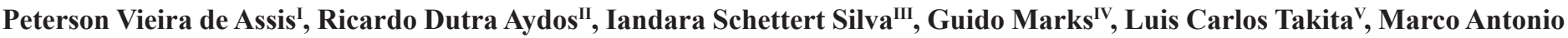 \\ Gonçalves $^{\mathrm{VI}}$, Rondon Tosta RamalhovII
}

\begin{abstract}
${ }^{I}$ Master, Health and Development, Postgraduate Program, Mato Grosso do Sul Federal University (UFMS), Campo Grande-MS, Brazil. Acquisition and interpretation of data.

"PhD, Associate Professor, Department of Surgery, UFMS, Campo Grande-MS, Brazil. Design of the study.

IIIPhD, Associate Professor, Department of Surgery, UFMS, Campo Grande-MS, Brazil. Helped with technical procedures.

${ }^{\text {IV }} \mathrm{PhD}$, Associate Professor, Department of Surgery, UFMS, Campo Grande -MS, Brazil. Critical revision, supervised all phases of the study.

${ }^{v} \mathrm{PhD}$, Assistant Professor, Department of Surgery, UFMS, Campo Grande-MS, Brazil. Histopathological examinations.

VIPhD, Department of Urology, UFMS, Campo Grande-MS, Brazil. Statistical analysis, critical revision.

${ }^{\mathrm{VII}}$ Master, Fellow PhD degree, Health and Development, Postgraduate Program, UFMS, Campo Grande-MS, Brazil. Histopathological examinations, manuscript writing.
\end{abstract}

\begin{abstract}
PURPOSE: To investigate the expression of FAS ligand (FASL) in ipsilateral and contralateral testicles of rats submitted to ischemia/ reperfusion.

METHODS: Wistar rats ( $\mathrm{n}=21)$ distributed into groups control (GC), $\mathrm{n}=5$, testicular exposure; ischemia (GI), ( $\mathrm{n}=8)$, Torsion in the left testicular Cord (TCT) for three hours followed by orchiectomy without distortion and orchietomy of the contralateral testicle after 24 hours; and reperfusion (GR), (n=8), left TCT for 3 hours and distortion and repositioning on the scrotum and bilateral orchiectomy after 24 hours. Quantification of the FASL expression by immune-histochemistry.

RESULTS: Statistical analysis showed similarity between GC and GI ( $>00.05)$, differences detected are concentrated on the GR $(p<0.05)$, increase in immunoexpression of FASL in the subgroups Right GR (406.8+-61.5) and Left GR (135.3 +-28.9) with significant predominance in the GR subgroup.
\end{abstract}

CONCLUSION: Ischemia/reperfusion increased the FASL expression significantly in contralateral testicles in GR, in rats.

Key words: Fas Ligand Protein. Spermatic Cord Torsion. Immunohistochemistry. Ischemia. Reperfusion Injury. Oxidative Stress. Rats. 


\section{Introduction}

Testicular Cord Torsion (TCT) results from the inadequate rotation of the testitcle around its longitudinal axis. It is a pathological condition that makes the testicles ischemic, and surgical intervention is usually necessary to re-establish blood flow ${ }^{1}$.

The treatment consists of surgical distortion and bilateral orchidopexia by transcrotal access, with orchiotomy indicated only for cases that clearly unviable that did not reperfund after surgical distortion, or with necrosis ${ }^{2}$.

In histological terms, the torsion seems to induce in the contralateral testicle a focal lesion in the seminiferous tubules, characterized by apoptosis and shedding of germinative cells, possibly mediated by cellular and humoral immune response ${ }^{3}$.

The contralateral testicular lesion from the torsion of the testicular cord is controversial. Some authors show that the unilateral testicular torsion causes contralateral testicular lesion ${ }^{4}$, while others show that there is no contralateral lesion ${ }^{5-7}$.

Several theories have been proposed in the atempt to explain the possible damage to the contralateral testicle, including autoimmune reaction, subclinical episodes of contralateral testicular torsion, release of acrosomic enzymes, vasomotor reflex, neuroendocrine response, subjacent defect in the spermatogenesis and intrinsic gonadal abnormality ${ }^{8,9}$.

The FAS ligand is a glucoprotein isoalted in $1994^{10}$. The FAS ligand protein (FASL) is an extrinsic activator of apoptosis that can be quantified by immuno-histochemistry. To determine the behavior of FASL, in experimental model, facing oxidative stress is important in the comprehension of the activation pathway of the apoptosis in the testicles submitted to the ischemia / reperfusion phenomenon.

In the testicle, the FAS system plays an important role in the apoptosis of germinative cells, after ischemia / reperfusion, stress and exposure of the testicle to toxic agents ${ }^{11,12}$.

In a study involving FAS and FAS ligand in the apoptosis of testicular germinative cells by zearalenone (ZEA), in rats, the authors concluded that the acute exposure of a nonsteroidal estrogenic mycotoxin (ZEA) induced the apoptosis in the germinative cells of male rats. This toxicity is parcially mediated through the modulation of the FAS and FASL system. However, while a maximum number of apoptotic cells and a peak in FAS expression were seen at 12 hours, the levels in FASL expression incresed in a time dependant form ${ }^{13}$.

In a study to quantify the FASL expression, using azoxymethane which induces oxidative stress in the gastric mucossa of Wistar rats, teh FASL protein was analyzed using the immuno-histochemical method, showing increase in its expression three hours after azoxymethane application ${ }^{14}$.

To determine the behavior of the FASL facing the oxidative stress in an experimental model is important in the comprehension of the activation pathway of the apoptosis, and it could explain what occurs in the contralateral testicle during TCT. The present study had the objective of evaluating the immunohistochemical expression of the apoptosis activating protein, FASL in ipsilateral and contralateral testicles of rats submitted to the ischemia/reperfusion phenomenon, by torsion of the unilateral testicular cord.

\section{Methods}

The research project was approved by the Ethics Committee in animal use of the Federal University of Mato Grosso do Sul (UFMS), under protocol number 317/2011.

A total of 21 rats (Rattus norvegicus albinus), male, of the Wistar lineage, having mean weight of $280 \pm 20$ grams, 70 days old, from the Animal Colony, Mato Grosso do Sul Federal University. The animals were kept in the experiment bioterium for seven days prior the procedures. Randomly, the animals were distributed into three groups: control (GC), $n=5$, only testicular exposure; ischemia (GI), $(n=8)$, torsion of the left testicular cord at $720^{\circ}$ for three hours followed by immediate orchiectomy of this testicle without distortion and orchiectomy of the contralateral testicle after 24 hours; and reperfusion $(\mathrm{GR}),(\mathrm{n}=8)$, torsion of the left testicular cord at $720^{\circ}$ for three hours followed by distortion and repositioning of the testicle in the scrotum and bilateral orchiectomy after 24 hours, subdividing each group into two subgroups: E (left-ipsilateral) and D (right-contralateral).

\section{Immunohistochemistry}

The immuno-histochemical processing (avidin-biotin complex, ABC technique) was made using the anti-FAS ligand primary antibody (termination epitope - N20, Santa Cruz ${ }^{\circledR}$, product SC834, lot A1812, dilution 1:100), secondary antibody Dako LSAB $^{\circledR}$ (product K0609, lot 10055833) and revealer of color DAB (diamino + benzidine) Dako ${ }^{\circledR}$ (product K3468, lot 10029914, dilution 1:10), with immunopositivity of the anti-FAS ligand biomarker expression, in brown coloring.

For the immune-histochemical quantification, 40 seminiferous tubules were selected, previously marked with software to avoid double counting. We counted the Sertoli 
cells with a 40x lens, identifying those with strongly positive immunoexpression for FAS ligand.

\section{Statistical analysis}

The software GraphPad Prism 5.0 was used for the statistical analyses. The sample distribution of the data was determined by the Kolomogorov-Smirnov normality test. The multiple comparisons were made using one-way ANOVA followed by the bicaudal " $t$ " Student post-test for the comparison of the left and right sides of each experimental group and Tukey post-test for the differences among the groups.

All of the statistical tests were made at a significance level of $5 \%$.

\section{Results}

The results obtained are presented in Table 1.

TABLE 1 - Mean count of Sertoli cells that expressed FAS ligand in the control, ischemia and reperfusion groups - 2012.

\begin{tabular}{lccc}
\hline \multirow{2}{*}{ Groups } & \multicolumn{2}{c}{ Testicles } \\
\cline { 2 - 4 } & Ipsilateral & Contralateral & $\boldsymbol{p}$ \\
\hline Control & $60.2 \pm 21.4^{\mathrm{a}}$ & $63.8 \pm 34.6^{\mathrm{c}}$ & 0.880 \\
Ischemia & $88.8 \pm 33.5$ & $68.8 \pm 35.3$ & 0.305 \\
Reperfusion & $135.3 \pm 28.9^{\mathrm{b}}$ & $406.8 \pm 61.5^{\mathrm{d}}$ & $<0.0001^{*}$ \\
\hline
\end{tabular}

$p$ related to the comparison of the FASL expression between the ipsilateral and contralateral testicles

a $p>0.05$ in relation to the ischemia group

$\mathrm{b} p<0.05$ in relation to the control and ischemia groups

$\mathrm{c} p>0.05$ in relation to the ischemia group

$\mathrm{d} p<0.0001$ in relation to the control and ischemia groups

The control group presented statistical equality $(\mathrm{p}=0.880)$ among the count in the left $(60.2 \pm 21.4)$ and right $(63.8 \pm 34.6)$ sides. The same was seen for the group which the tissue ischemia was made ( $\mathrm{p}=0.305)$, with the left and right sides statistically similar ( $88.8 \pm 33.5$ and $68.8 \pm 35.3$, respectively).

In the reperfusion group, however, there was significant difference between the sides $(\mathrm{p}<0.0001)$, with a lower number of Sertoli cells expressing the FAS ligand on the left side $(135.3 \pm$ 28.9) than the right $(406.8 \pm 61.5)$ (Figure 1).

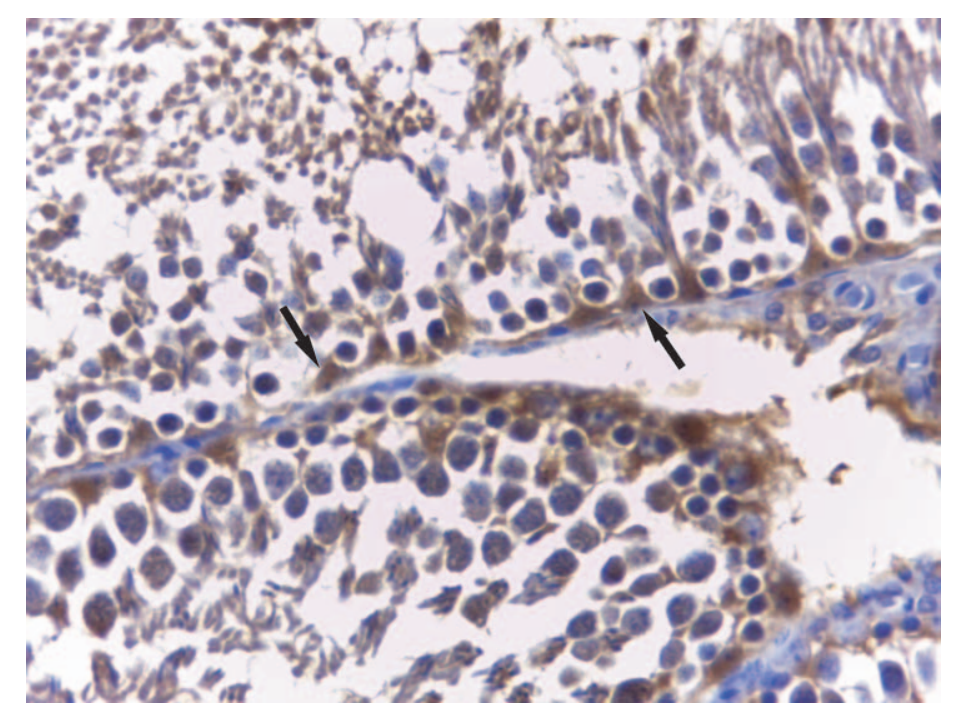

FIGURE 1 - Photomicrography of seminiferous tubules, x400 increase, showing the Sertoli cells with strongly positive expression of FASL, in brown.

In the statistical analysis of the results from the epsilateral testicles between the studied groups, we observed a presence of significant differences among the tested procedures $(p=0.0008)$. The "control" and "ischemia" groups were similar ( $p>0.05)$, been that the differences detected were found in the "reperfusion" group $(p<0.05)$ according to the representation in Figure 2.

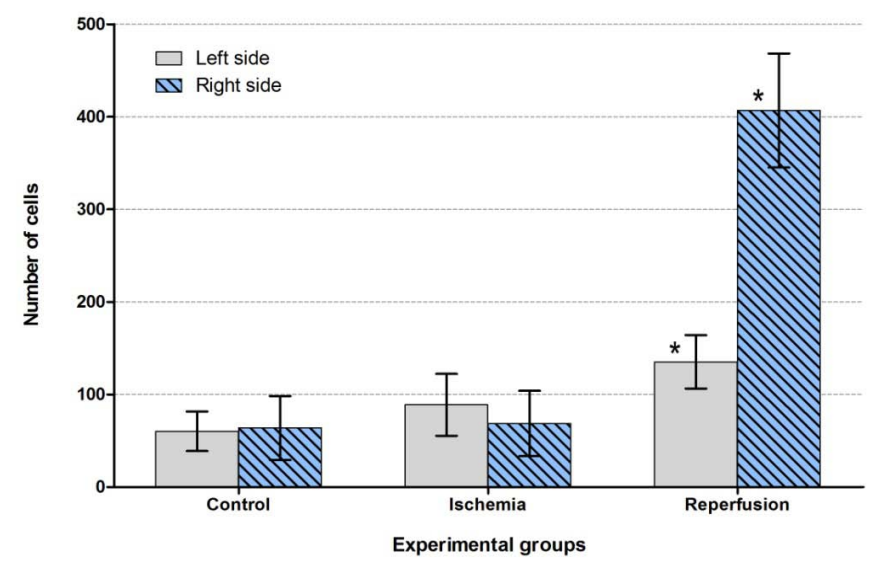

FIGURE 2 - Mean count of Sertoli cells that expressed FAS ligand, according to each side and experimental group.

In the contralateral testicles statistically significant differences were seen among the procedures $(p<0.0001)$. Although, the "control" and "ischemia" groups were similar $(p>0.05)$, and the differences detected were concentrated in the "reperfusion" group $(\mathrm{p}<0.0001)$. 


\section{Discussion}

The topic of torsion of the testicular cord has been widely researched under several perspectives like the effect of ischemia on the testicular parenchyma, the phenomenon of ischemia/ reperfusion, activation of apoptosis as potential influence in the extension of the tissue lesion, involvement of the FAS system in the induction of apoptosis in germinative cells, the damage on the contralateral testicle and the means to avoid or decrease the effects of the torsion. The diverse methodology in the studies has as consequence varied and diverging results ${ }^{15-18}$.

In relation to the degree of TCT that can lead to testicular lesion, there are studies that show after a torsion of $720^{\circ}$, there was residual blood flow, varying between $0 \%{ }^{15}, 8.8 \%{ }^{19}$ and $38.3 \%{ }^{20}$ and reaching after distortion, statistically similar values top the pre-torsion blood flow, varying from 24 hours $^{15}$, and after 30 days ${ }^{19,20}$, this fact can try to explain the lesion in the contralateral testicle.

Most of the revised studies in literature that have studied the FASL expression in rats used toxic substances like zearalenone $^{13}$ and azoxymethane ${ }^{14}$, differently from this study, where we studied the FASL expression through the induction of the ischemia/reperfusion phenomenon by torsion / distortion of the testicular cord in rats. We observed that the oxidative stress caused by toxic substances as well as the method used in this study induced the increase in FASL expression.

The FASL expression in the testicles of rats, by points in time (three, six, 12, 24 and 48 hours), after application of zearalenone, showed stable increase until 48 hours ${ }^{13}$. In another study with rats using clamping of testicular arteries and veins using vascular clamps for 60 minutes and reperfusion by points in time (zero, 12, 24 and 48 hours), Sertoli cells were identified positive for FASL in any point in time, with slight elevation at 24 hours of reperfusion ${ }^{17}$.

There expected was that there was a higher number of Sertoli cells expressing FASL, in the ipsilateral testicles of GR; however, this experimental study showed important increase in Sertoli cells expressing FASL in the contralateral testicles of GR, warning on the possibility of reperfusion along with the increase of FASL expression be the cause of damage in these testicles, further research is necessary to confirm and justify these results. Confirming these results in other studies, this could be another theory to explain the lesion on contralateral testicle.

Further research is needed to elucidate when is the right moment to intervene, keeping or not the testicle that suffered torsion in the cord, therefore protecting the spermatogenesis of the patients, and thus their reproductive capacity.

\section{Conclusion}

The expression of FAS ligand increased in the ipsilateral and contralateral testicles of the reperfusion group, after three hours of ischemia and 24 hours of reperfusion, more significantly so in the contralateral testicles.

\section{References}

1. Williamson RC. The continuing conundrum of testicular torsion. $\mathrm{Br}$ J Surg. 1985;72(7):509-10.

2. Jesus LE. Acute scrotum. Rev Col Bras Cir. 2000;27(4):271-8.

3. Rodriguez MG, Rival C, Theas MS, Lustig L. Immunohistopathology of rats undergoing experimental torsion of the spermatic cord. Asian J Androl. 2006;8(5):576-83.

4. Mogilner JG, Lurie M, Coran AG, Nativ O, Shiloni E, Sukhotnik I. Effect of diclofenac on germ cell apoptosis following testicular ischemia-reperfusion injury in a rat. Pediatr Surg Int. 2006;22:99105.

5. Bozlu M, Coşkun B, Cayan S, Acar D, Aktaş S, Ulusoy E, Akbay E. Inhibition of poly (adenosine diphosphate-ribose) polymerase decreases long-term histologic damage in testicular ischemiareperfusion injury. Urology. 2004;63(4):791-5.

6. Duru M, Gergerlioglu HS, Akaydin Y, Sogut S. The protective role of erdosteine on testicular tissue after testicular torsion and detorsion. Mol Cell Biochem. 2005;280(1-2):193-9.

7. Salmasi AH, Beheshtian A, Payabvash S, Demehri S, Ebrahimkhani MR, Karimzadegan M, Bahadori M, Pasalar P, Dehpour AR. Effect of morphine on ischemia-reperfusion injury: experimental study in testicular torsion rat model. Urology. 2005;66(6):1338-42.

8. Nguyen L, Lievano G, Ghosh L, Radhakrishnan J, Fornell L, John E. Effect of unilateral testicular torsion on blood flow and histology of contralateral testes. J Pediatr Surg. 1999;34(5):680-3.

9. Visser AJ, Heyns CF. Testicular function after torsion of the spermatic cord. BJU Int. 2003;92(3):200-3.

10. Suda T, Nagata S. Purification and characterization of the Fas-ligand that induces apoptosis. J Exp Med. 1994;179(3):873-9.

11. Koji T, Hishikawa Y. Germ cell apoptosis and its molecular trigger in mouse testes. Arch of Histol Cytol. 2003;66:1-16.

12. Boekelheide K. Mechanisms of toxic damage to spermatogenesis. J Natl Cancer Inst Monogr. 2005;(34):6-8.

13. Jee YH, Noh EM, Cho ES, Son HY. Involvement of the Fas and Fas ligand in testicular germ cell apoptosis by zearalenone in rat. J Vet Sci. 2010;11(2):115-9.

14. Bussuan LAM, Fagundes DJ, Marks G, Bussuan PM, Teruya R. The role of Fas ligand protein in the oxidative stress induced by azoxymethane on crypt colon of rats. Acta Cir Bras. 2010;25:501-6.

15. Turner TT. Acute experimental testicular torsion. No effect on the contralateral testis. J Androl. 1985;6(1):65-72.

16. Turner TT, Tung KS, Tomomasa H, Wilson LW. Acute testicular ischemia results in germ cell-specific apoptosis in the rat. Biol Reprod. 1997;57(6):1267-74.

17. Koji T, Hishikawa Y, Ando H, Nakanishi Y, Kobayashi N. Expression of Fas and Fas ligand in normal and ischemiareperfusion testes: Involvement of the Fas system in the induction of germ cells apoptosis in the damaged mouse testis. Biol Reprod. 2001;64(3):946-54. 
18. Urt Filho A, Inouye CM, Pontes JCDV, Silva ACBA, Silva GVR, Santos CHM. Propofol effects on the morphology of rat testes subjected to testicular ischemia $\neg$ reperfusion. Acta Cir Bras. 2012;27(2):172-8.

19. Becker EJ, Prillaman HM, Turner TT. Microvascular blood flow is altered after repair of testicular torsion in the rat. J Urol. 1997;157(4):1493-8.

20. Palmer JS, Plzak LF, Cromie WJ. Comparison of blood flow and histological changes in rat models of testicular ischemia. J Urol. 1997;156(3):1138-40

\section{Correspondence:}

Ricardo Dutra Aydos

Avenida Senador Felinto Muller, s/n - Cidade Universitária 79080-190 Campo Grande - MS Brasil

ricadoaydos@gmail.com

Received: March 18, 2013

Review: May 17, 2013

Accepted: June 19, 2013

Conflict of interest: none

Financial source: none

${ }^{1}$ Research performed at Health and Development, Postgraduate Program, Federal University of Mato Grosso do Sul (UFMS), Campo Grande, Brazil. Part of Master degree thesis. Tutor: Prof. Ricardo Dutra Aydos. 